\title{
Detection of local defects in textile webs using Gabor filters
}

\author{
Jaume Escofet \\ Escola Universitària d'Optica i Optometria \\ de Terrassa \\ Departament d'Optica i Optometria \\ Edifici TR8, Campus de Terrassa \\ 08222 Terrassa, Barcelona, Spain \\ E-mail: jescofet@oo.upc.es \\ Rafael Navarro \\ Instituto de Optica \\ Consejo Superior de Investigaciones \\ Científicas \\ C/Serrano 121 \\ 28006 Madrid, Spain \\ María S. Millán \\ Josep Pladellorens \\ Escola Universitària d'Optica i Optometria \\ de Terrassa \\ Departament d'Òptica i Optometria \\ Edifici TR8, Campus de Terrassa \\ 08222 Terrassa, Barcelona, Spain
}

\begin{abstract}
A method of image analysis is proposed for detection of local defects in materials with periodic regular texture. A general improvement and enlargement of vision system capabilities for versatility, full automatism, computational efficiency, and robustness in their application to the industrial inspection of periodic textured materials is pursued. In the proposed method, a multiscale and multiorientation Gabor filter scheme that imitates the early human vision process is applied to the sample under inspection. The designed algorithm automatically segments defects from the regular texture. A variety of examples of fabric inspection are presented. In all of them defects are successfully segmented from the texture background. (c) 1998 Society of Photo-Optical Instrumentation Engineers. [S0091-3286(98)01408-1]
\end{abstract}

Subject terms: Gabor functions; image analysis; web inspection; defect detection.

Paper 17107 received Oct. 15, 1997; revised manuscript received March 16 , 1998; accepted for publication March 17, 1998. This paper is a revision of a paper presented at the EUROPTO Conference on Lasers, Optics, and Vision for Productivity in Manufacturing I, June 1996, Besançon, France. The paper presented there appears (unrefereed) in Proc. SPIE Vol. 2785.

\section{Introduction}

The interest in reliable, automatic systems for visual inspection of industrial materials such as textile webs, paper, or wood requires the development of image segmentation techniques based on texture analysis. The surfaces of such materials display complex patterns that appear visually regular on a large scale. In a local analysis, however, the texture components may vary in their intensity distribution, pattern size, and pattern shape. These local variations make inspection difficult.

Fourier-domain-based techniques are particularly suitable for materials that exhibit a high degree of periodicity (e.g., most textile webs). The angular and radial analysis of the Fourier transform of a web image provide valuable information for characterizing carpet patterns ${ }^{1}$ or common fabrics. ${ }^{2}$ Other related operations, such as autocorrelation of a web image, have been proposed in Ref. 1 and used in Ref. 2 to explore the yarn spacing in the weft and warp directions. Ciamberlini et al. ${ }^{3}$ describe an optical method using Fourier transform and spatial filtering to reveal defects in textile materials in real time. Recently, Millán and Escofet ${ }^{4}$ have proposed Fourier-domain-based angular correlation for pattern recognition of quasiperiodic textures. It has been applied to web inspection for pattern identification and classification, and also for the detection and characterization of defects that cause an overall distortion of the basic structure of the material, such as shrinking and abrasion.

When defects only alter a small area of the image of the material under inspection, they are called local defects. Fourier analysis does not provide, in general, enough infor- mation to detect local defects. Methods that can localize and analyze features in the spatial as well as in the frequency domain are convenient for detecting local defects. Wavelet transforms, used as multiresolution spectral filters, provide both frequency and spatial local information about an image. In fact, different wavelet bases have been used to develop applications of image analysis to local-defect detection in woven fabrics. ${ }^{5-7}$ In a preliminary work, ${ }^{7}$ we evidenced the feasibility of using Gabor filters ${ }^{8}$ to the inspection of local defects in fabrics.

A Gabor filter consists of a sinusoid of a given frequency and orientation, modulated by a Gaussian envelope. This Gaussian envelope provides spatial localization. A good reason for the use of Gabor filters is their relationship with current models of early vision in primates; in addition they have optimal localization in the space and frequency domains ${ }^{9}$ with an efficient implementation in both domains. ${ }^{10}$ The human eye is a highly efficient visual system and a robust pattern and texture analyzer. Gabor and related wavelets used in visual modeling have been successfully applied to a large variety of early vision tasks. ${ }^{10}$ Specifically, the problem of detecting local defects in a surface can be related to texture segmentation when either the material, the defect, or both are textured, as in textile webs. Several authors have proposed different approaches based on Gabor filters for texture segmentation ${ }^{11-15}$ and for object detection. ${ }^{16}$

Here we present a new method for automatic detection of local defects in a regular texture, based on a multiscale and multiorientation Gabor scheme. This scheme imitates the visual coding in the early stages of the human visual 
system and was proposed as an image representation model. ${ }^{17}$ Its usefulness has been demonstrated through a variety of applications involving local multiscale processing and texture analysis ${ }^{15}$ and synthesis. ${ }^{18}$ According to this Gabor scheme, we build an algorithm that uses the filtered images of the textured material under inspection in order to locate and isolate their possible defects.

We have successfully applied the method to detect a variety of typical defects in woven fabrics of different structure. In this paper, we present and discuss some examples.

\section{Method}

In this section we briefly review the multiscale and multiorientation Gabor scheme for feature extraction, and then describe the procedure designed to segment defects from the background (regular texture).

\subsection{Multiscale and Multiorientation Gabor Scheme}

We use the model proposed in Ref. 17, which seeks to simulate schematically the early visual coding in humans by applying a set of $4 \times 4$ Gabor filters to digital images. This scheme performs a logarithmic-polar sampling of the frequency spectrum of the image. The sampling yields four frequency levels distributed in octaves and four orientations (horizontal, vertical, and the two diagonals). A low-pass residual channel (LPR channel), at the center, covers the very low frequencies around dc. The general description of the model we consider is contained in Sec. 2 of Ref. 18. In particular we recall that a 2-D Gabor function in the spatial domain of coordinates $(x, y)$ is given by

$$
\begin{aligned}
g(x, y)_{p q}= & \exp \left[-\frac{\pi}{a_{p}^{2}}\left(x^{2}+y^{2}\right)\right] \\
& \times \exp \left[i 2 \pi f_{p}\left(x \cos \theta_{q}+y \sin \theta_{q}\right)\right],
\end{aligned}
$$

where the first factor represents the Gaussian envelope with bandwidth determined by the parameter $a_{p}$, and the second factor is a complex sinusoid. The parameters $f_{p}$ and $\theta_{q}$, with $p, q=1, \ldots, 4$, represent respectively the frequency and the orientation of the $p q$ channel. For an input image $i(x, y)$ and a Gabor filter given by the complex function $g_{p q}(x, y)$ of Eq. (1), the magnitude of the filtered image, $\left|i_{p q}(x, y)\right|$, can be computed as

$\left|i_{p q}(x, y)\right|=\left\{\left[g_{p q}^{e}(x, y) * i(x, y)\right]^{2}+\left[g_{p q}^{o}(x, y) * i(x, y)\right]^{2}\right\}^{1 / 2}$,

where the symbol * denotes a 2-D convolution, and $g_{p q}^{e}$ and $g_{p q}^{o}$ represent the real (even) and the imaginary (odd) parts, respectively, of the Gabor filter $g_{p q}(x, y)$.

As described in Ref. 17, an appropriate filter design with small convolution masks allows an efficient implementation of Gabor filters in the spatial domain. Moreover, the pyramidal structure and the symmetries of the scheme simplify its application by using self-similar Gabor wavelets. Instead of scaling the wavelet, it is preferable to undersample the input $i(x, y)$ by a factor $2^{4-p}$ corresponding to the channel of frequency $f_{p}$. The input is conveniently convolved with a low-pass filter (e.g., a cubic B spline ${ }^{19}$ ) before subsampling in order to remove the high-frequency terms and avoid aliasing artifacts. For the convolution in the spatial domain, we use two masks of $9 \times 9$ pixels that represent the even and the odd parts of the Gabor filter $g_{p q}(x, y)$. The magnitudes $\left|i_{p q}(x, y)\right|$ of the $4 \times 4$ channels will constitute the input of the segmentation algorithm.

\subsection{Segmentation}

This section describes the procedure we propose for defect detection in regular textures. There is an extensive literature suggesting that Gabor channels are good descriptors of texture. ${ }^{7,10-12,14,15,18}$ A visible defect will cause a local change of visual texture. If the proposed Gabor channels are able to describe texture accurately, then we can exploit this fact to segment the image automatically and detect relevant defects visually. The presented method must meet three requirements. Firstly, we have to enhance changes in the descriptors, which may correspond to a fault in such a way that a binarization makes possible the segmentation of defective areas from the textured background. Secondly, the process must integrate faults captured at different orientations and resolution levels of the Gabor filters into a single binary map with the locations of defects as the output. Thirdly, the procedure must be automatic, robust, and versatile, easily adaptable to a variety of regular textures of different materials. This third aspect entails that we will not introduce key parameters, which may require specific adjustments of parameters or procedures to a particular kind of defect or texture. In this way, we will avoid problems of overtraining or undertraining that frequently appear when optimizing a given method with a limited, incomplete set of training samples.

Figure 1(a) shows a schematic diagram of the procedure. It starts with an image of the sample to inspect: $t(x, y)$. The set of Gabor filters described in Sec. 2.1 is applied to the input image $t(x, y)$ to give the moduli of the set of $4 \times 4$ filtered complex images, $\left|t_{p q}(x, y)\right|$. The low-pass residual image, $t_{\mathrm{LPR}}(x, y)$ is obtained by subsampling the input $t(x, y)$ four times by a factor 2 (including a convolution with the low-pass filter cubic B spline before each subsampling).

In the second step, our texture descriptors are obtained by expressing the filtered images $\left|t_{p q}(x, y)\right|$ in contrast units. This can be accomplished by dividing every filtered image $\left|t_{p q}(x, y)\right|, p, q=1, \ldots, 4$, by the low-pass residual image $t_{\mathrm{LPR}}$. Thus, the set $\{p, q\}$ of features $T_{p q}(x, y)$ is given for each pixel $(x, y)$ by the expression

$T_{p q}(x, y)=\frac{\left|t_{p q}(x, y)\right|}{t_{\mathrm{LPR}}\left(x^{\prime}, y^{\prime}\right)}$,

with (see Fig. 2)

$x^{\prime}=1+I\left(\frac{x-1}{2^{p}}\right), \quad y^{\prime}=1+I\left(\frac{y-1}{2^{p}}\right)$,

where function $I(z)$ means the integer part of the argument $z$. 

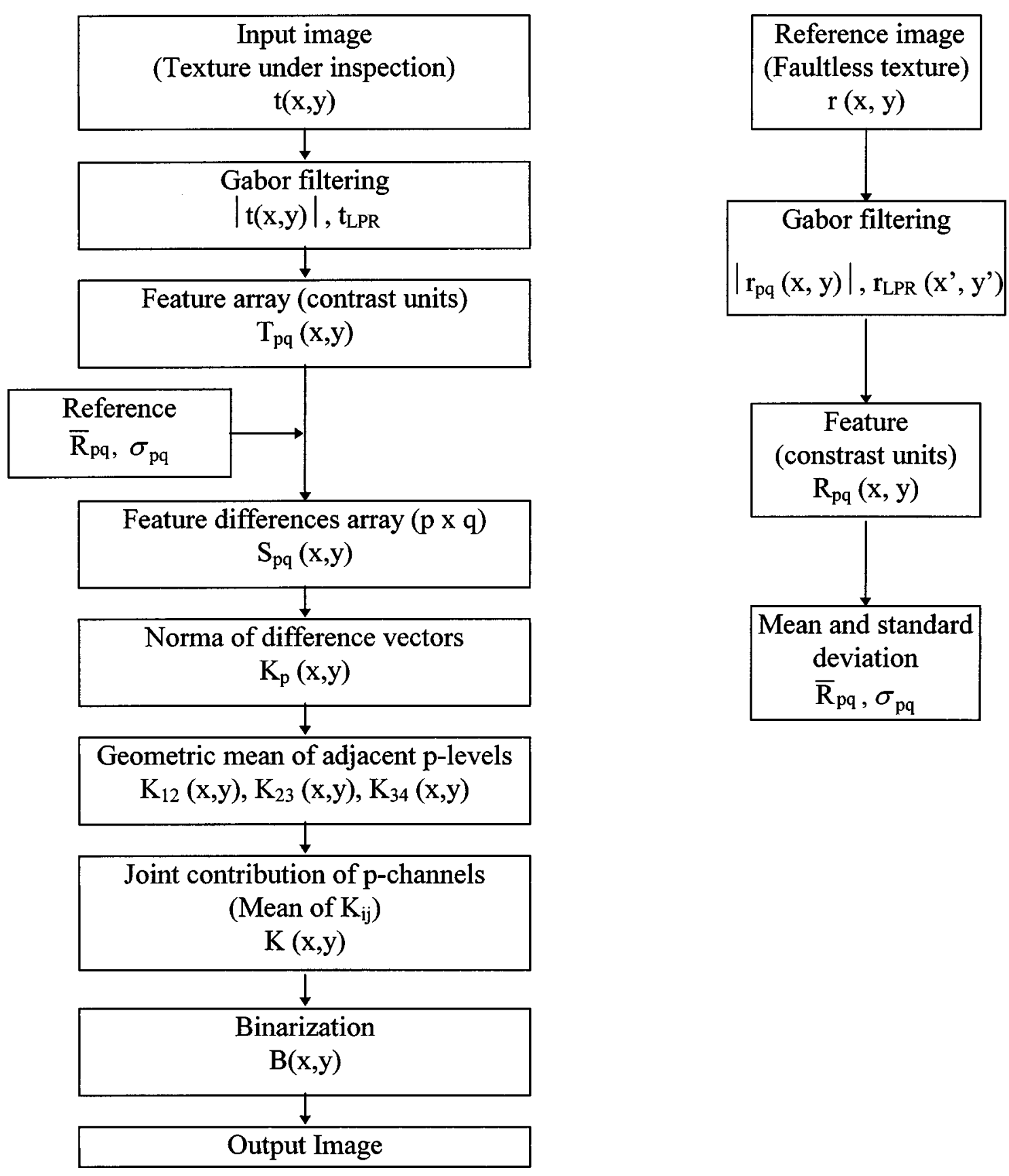

Fig. 1 Schematic diagram of the segmentation procedure: (a) main diagram; (b) detail of the part of the procedure applied to a faultless sample (prototype), which provides the reference entry to the main diagram (a) (on the left).

Before analyzing the texture to be tested, we first apply the same procedure to a prototype defect-free sample $r(x, y)$ and store the mean and standard deviation of the histograms of each feature [the block on the left in Fig. 1(a), sketched in detail in Figure 1(b)]. We assume that both the image $t(x, y)$ of the texture under inspection and the image $r(x, y)$ of the faultless reference texture are acquired under the same experimental conditions of scale, orientation, and resolution. In Fig. 1(b), the filtered images in absolute value, $\left|r_{p q}(x, y)\right|$, are again converted to contrast units, by dividing by the corresponding LPR image $r_{\mathrm{LPR}}\left(x^{\prime}, y^{\prime}\right)$ :
$R_{p q}(x, y)=\frac{\left|r_{p q}(x, y)\right|}{r_{\mathrm{LPR}}\left(x^{\prime}, y^{\prime}\right)}$,

in the same way as in Eq. (3). The mean value (over all the pixels) of each $R_{p q}$ and the standard deviation $\sigma_{p q}$ are calculated by the standard expressions

$\bar{R}_{p q}=\frac{1}{N_{p}^{2}} \sum_{x=1}^{N_{p}} \sum_{y=1}^{N_{p}} R_{p q}(x, y)$, 


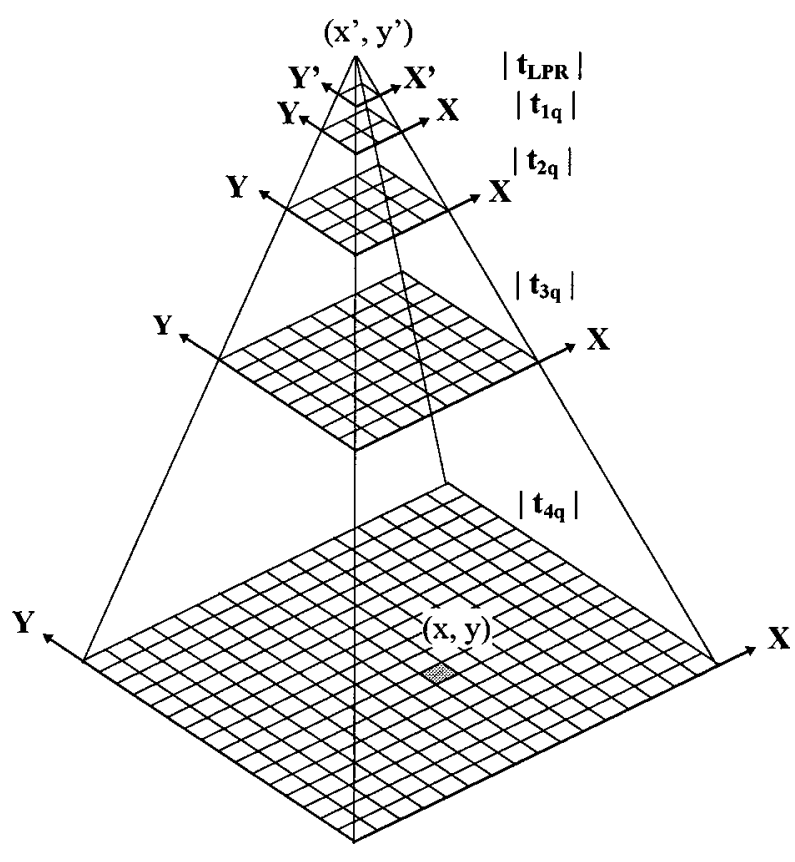

Fig. 2 Partial representation of the pyramid distribution used to express the filtered images $\left|t_{p q}(x, y)\right|$ in contrast units by dividing by the low-pass residual $t_{\mathrm{LPR}}$.

$\sigma_{p q}=\left\{\frac{\sum_{x=1}^{N_{p}} \Sigma_{y=1}^{N_{p}}\left[R_{p q}(x, y)-\bar{R}_{p q}\right]^{2}}{N_{p}^{2}}\right\}^{1 / 2}$,

where $N_{p}^{2}$ is the number of pixels of the filtered image at the resolution level $p$. The two sets of sixteen $(4 \times 4)$ values $\left\{\bar{R}_{p q}\right\}$ and $\left\{\sigma_{p q}\right\}$ are the reference entry to the main procedure on the left of the scheme in Fig. 1(a).

The next step is to compare, for each pixel or location, the features of the sample under study with those of the reference. The closer the values, the higher the likelihood of the sample coinciding with the prototype, and conversely, the larger the difference, the higher the probability of there being a defect. Thus, we calculate, for each level $p$ and orientation $q$, the magnitude of the difference between features of the sample under analysis and the mean of the prototype

$d_{p q}(x, y)=\left|T_{p q}(x, y)-\bar{R}_{p q}\right|$.

In order to reduce noise, for each pixel we set to zero those differences $d_{p q}(x, y)$ below a threshold, i.e., for those values of the sample with a high likelihood of being like the prototype. We consider a standard thresholding operation given by the expression

$S_{p q}(x, y)= \begin{cases}d_{p q}(x, y) & \text { if } d_{p q}(x, y) \geqslant \tau \sigma_{p q} \\ 0 & \text { otherwise, }\end{cases}$

where the threshold is proportional to the standard deviation $\sigma_{p q}$ calculated from the reference feature array $R_{p q}(x, y)$. We take a fairly standard constant value $\tau=3$ according to a low-risk criterion: only points with differences above three times the standard deviation are eligible as defects, which strongly reduces the probability of misclassifying points of the background (regular texture) as faulty areas. The resulting array of the thresholded feature differences is represented by $S_{p q}(x, y)$ in the diagram in Fig. 1(a).

For each scale level $p$ and for every pixel $(x, y)$, a vector of four components, $\mathbf{S}_{p}^{x y}=\left\{\left(S_{p}^{x y}\right)_{q}\right\}$ with $q=1, \ldots, 4$, can be built. Each component of the vector $\mathbf{S}_{p}^{x y}$ is defined by $\left(\mathbf{S}_{p}^{x y}\right)_{q} \equiv S_{p q}(x, y)$ and coincides with the thresholded feature difference of pixel $(x, y)$ at the scale level $p$ and orientation $q$. In the next stage an array $K_{p}(x, y)$ is calculated for each scale level $p$ with the norm of vectors $\mathbf{S}_{p}^{x y}$, that is,

$K_{p}(x, y)=\left\|\mathbf{S}_{p}^{x y}\right\|=\left\{\sum_{q=1}^{4}\left[S_{p q}(x, y)\right]^{2}\right\}^{1 / 2}$.

The definition of $K_{p}$, i.e., the norm of the featuredifference vector, is a common metric used in standard clustering algorithms for segmentation. According to Eq. (10), the array $K_{p}(x, y)$ concentrates the information on the likely defective areas obtained in the four orientations $q=1, \ldots, 4$ in a single array for the scale level $p$. Thus, the result of this stage is a set of four images $K_{p}(x, y)$ with $p=1, \ldots, 4$.

In the next two stages we combine the information coming from the four different resolution levels $p$. To this end the decompressed version of each array $K_{p}(x, y)$ is prepared. In order to avoid false alarms, we consider that a defect must appear in at least two adjacent resolution levels. As a simple way to implement a logic "and," assuming that $K_{p}(x, y)$ is proportional to the probability of there being a defect, we then calculate the geometric means of every pair of adjacent levels by the formulas:

$K_{12}(x, y)=\left[K_{1}(x, y) K_{2}(x, y)\right]^{1 / 2}$,

$K_{23}(x, y)=\left[K_{2}(x, y) K_{3}(x, y)\right]^{1 / 2}$,

$K_{34}(x, y)=\left[K_{3}(x, y) K_{4}(x, y)\right]^{1 / 2}$.

This operation reduces false alarms yet preserves most of the defective areas. Now we combine the resulting $K_{12}(x, y), K_{23}(x, y)$, and $K_{34}(x, y)$ in a logic "or," simply as the arithmetic mean, to allow for defects detected at different scales:

$K(x, y)=\frac{1}{3}\left[K_{12}(x, y)+K_{23}(x, y)+K_{34}(x, y)\right]$.

The array $K(x, y)$ contains the joint contribution of the sixteen $p q$ channels.

The last stage is the binarization of $K(x, y)$ to provide an image $B(x, y)$ where local defects (objects) appear segmented from the regular texture (background). This is achieved by thresholding $K(x, y)$. Values below the threshold are considered as belonging to the background, and values above the threshold are considered as belonging to defective areas. 
This threshold value is not critical and can be estimated in different ways. One possible way is to calibrate the system at the beginning of the process by applying the procedure to an additional piece of faultless texture whose image would be the input image $t_{0}(x, y)$. In this case the obtained array $K_{0}(x, y)$ should contain very low values. An estimation of the threshold $U$ as $U=\bar{K}_{0}+\rho \sigma_{0}$, with $\bar{K}_{0}$ being the mean value of $K_{0}(x, y), \sigma_{0}$ its standard deviation, and $\rho$ a standard constant of value $\rho=3$, provides an appropriate threshold value for binarization. Alternatively, a simpler way is to calculate $U=(\rho / 16) \Sigma_{p} \Sigma_{q} \sigma_{p q}$, which is proportional to the mean value of the sixteen standard deviations $\sigma_{p q}$ with a constant of proportionality equal to a standard value, for example, $\rho=3$. An opening operation with a small mask of $3 \times 3$ pixels helps to remove the remaining isolated noisy points from the binary output image $B(x, y)$.

\section{Results}

We have applied the process described in Sec. 2 to a variety of textile webs with different structures (plain, twill, etc.) and with yarns of different colors affected by common local defects. These defects are caused by missing or broken yarns or by changes in tension during production in the loom. The defects display a variety of shapes: line, spot, band, ladder, hole, etc. In this section we show the results with representative examples chosen from among those mentioned. The examples shown below were carried out using a Pulnix TM-765 camera and a MVP-AT Matrox framegrabber for image acquisition in a Pentium PC environment. The implementation of the algorithm involved Matlab tools.

For industrial application the inspection unit should adapt to the conditions of each particular case. The imaging hardware may consist of either one moving camera or several systems working in parallel for time saving, depending on the surface to be inspected and the speed requirements. In practice, needs can be very different. After the loom, the fabric usually passes through a checking machine where is inspected visually. When a defect is detected, a person decides either to mend it manually (if possible), or to record its location on a form, or even to cut the fabric from side to side, depending on how severe the defect is. The quality criteria applied in this checking machine are strongly dependent on the type of fabric, the manufacturer's standards, etc., and therefore the time taken at the checking machine also varies. We consider that there is generally enough spare time to apply our method of defect detection between the loom and the checking machine.

Before applying the algorithm, it is important to fix the acquisition conditions, not only in terms of uniformity but also in terms of scale and resolution. We consider the maximum frequency $f_{\max }=f_{4}=1 / 4$ yarn/pixel. This means that a woven yarn is digitized into four pixels on average. If the yarns in the weft and warp directions are of different thickness, the camera is adjusted to fit the thinner yarn to four pixels. In our experiments this adjustment was made manually. Adjustment of lightness and scale is reasonably easy and only needs to be done once unless we change the web. The images of the textile samples we analyzed are 256 $\times 256$ pixels in size.
Figure 3(a) shows a sample of twill fabric containing some faults. The yarns in the warp are of a different color from the yarns in the weft. The defect appears as aligned spots, although some isolated spots can also be found. Our algorithm for defect detection is applied to the image in Fig. 3(a), which is taken as the entry $t(x, y)$. Figures 3(b) to $3(\mathrm{e})$ show the decompressed versions of the arrays $K_{p}(x, y)$ for resolution levels $p=4, \ldots, 1$. Figure 3(f) is the image $K(x, y)$ with the joint contribution of all the $p q$ channels, and Fig. $3(\mathrm{~g})$ is the binary image $B(x, y)$, which is the thresholded binary version of $K(x, y)$ and constitutes the output image. It can be seen that both the aligned and the isolated defective spots are correctly segmented from the background in Fig. 3(g).

An interesting case is shown in Fig. 4. A sample of twill fabric contains a faulty band in the central part of the image [Fig. 4(a)]. The defect is called a thin place, and is caused by a lower density of filling yarns in this band. Figures 4(b) to 4(e) are again the decompressed $K_{p}(x, y)$ with $p=4, \ldots, 1$. Figure $4(\mathrm{f})$ is the array $K(x, y)$ with the joint contribution of channels, and Fig. $4(\mathrm{~g})$ is the final binarized image $B(x, y)$. Although the faulty band is clearly segmented in the final result, in this case the single resolution level $p=3$ [Fig. 4(c)] alone provides a better intermediate result. In this example, channel $p=3$ clearly provides the best tuning of the defect among the four resolution channels. The later operations (multiplication and addition), designed for the sake of automatism and robustness of method, to reduce noise and to integrate information from the four resolution channels, have the drawback of mixing channels that are very well tuned with the defect with others having no information. As a result, the quality of the segmentation is not so good as it could be if we chose the best channel alone. However, with this mixing procedure we gain robustness. The benefits of high robustness and automatism, regardless of the type of web or defect, are much more important than a perfect segmentation.

In the remaining figures we present (a) the input image of a fabric to inspect $t(x, y)$, together with (b) an image of the joint contribution channels $K(x, y)$ and (c) the final output image $B(x, y)$. Figures 5 and 6 correspond to twill fabrics with defects along a line: a missing yarn (mispick) and a double yarn, respectively. In both cases the output images contain the defects correctly discriminated from the background. Figures 7 and 8 correspond to twill samples with defects in a dotted distribution: several broken yarns and a down heddle defect respectively. The broken yarns are correctly segmented in Fig. 7(b). The small size of the defects in the down heddle defect makes for difficulty in detecting some dots and discriminating them from the background. Careful observation of the array $K(x, y)$ with the joint contribution of channels in Fig. 8(b) allows us to locate all the faults. The result of the final opening operation is, in this case, that some points are removed [Fig. 8(c)]. However, more than $50 \%$ of point defects (8 out of 15) are detected by applying the general method.

The plain fabric in Fig. 9(a) has two spots of very different intensity. The spots are quite big in comparison with previous dotted defects. Both spots are successfully segmented in Fig. 9(b) and 9(c). 


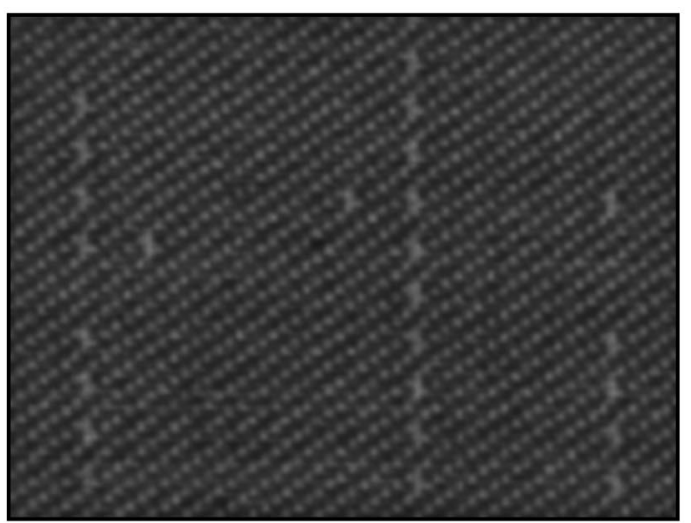

(a)

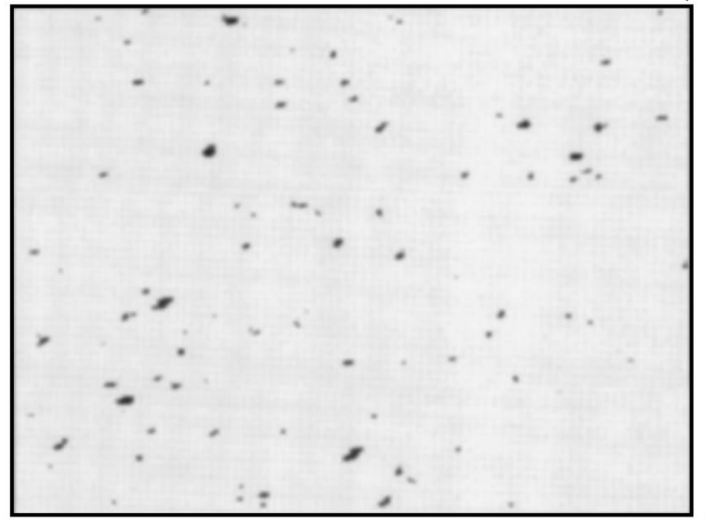

(b)

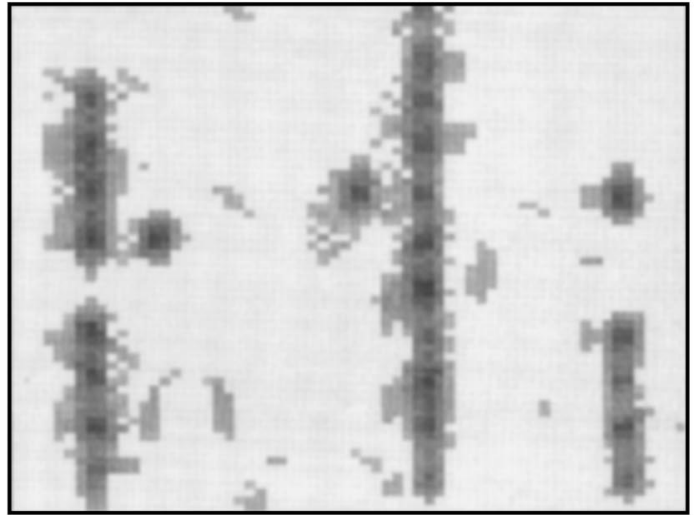

(d)

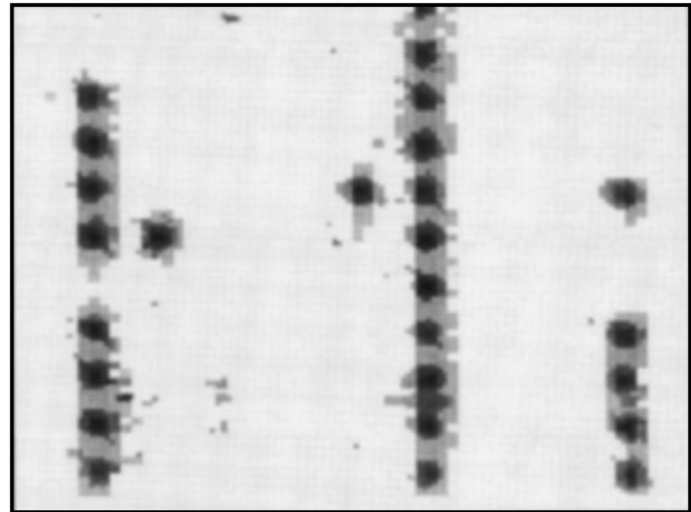

(f)

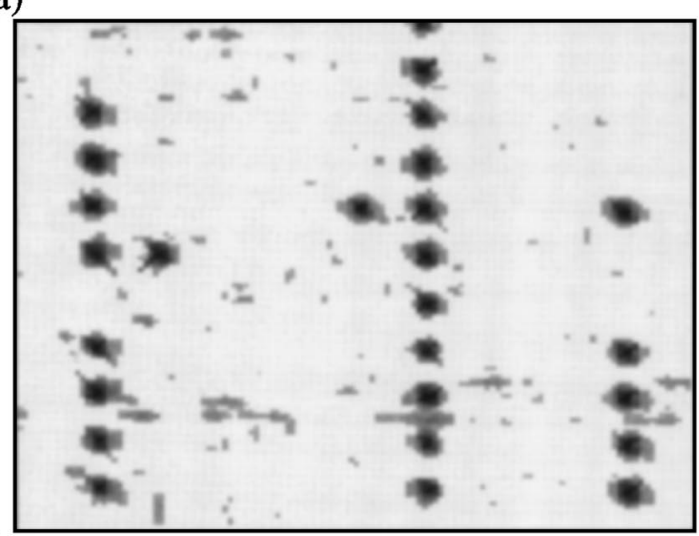

(c)

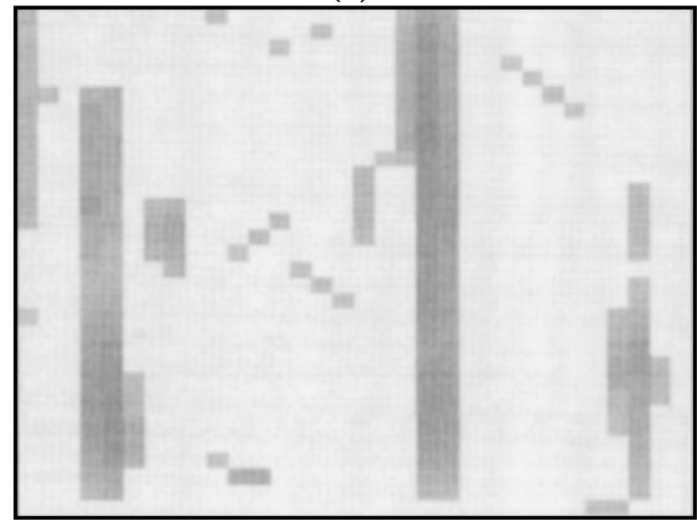

(e)

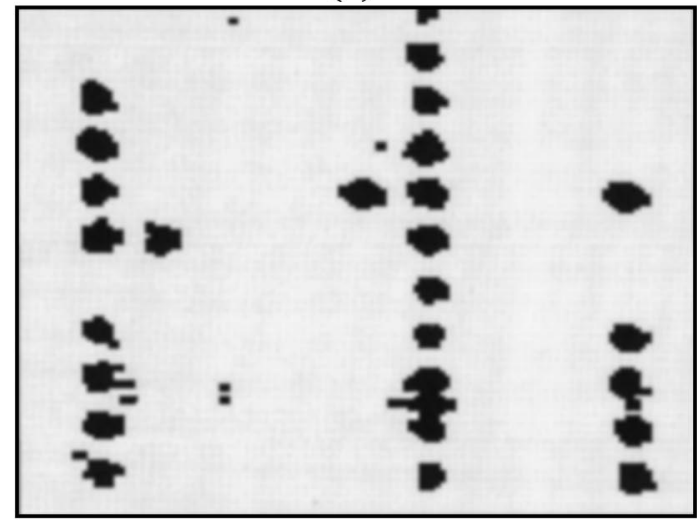

(g)

Fig. 3 (a) Faulty twill fabric (multiple threads broken); (b) to (e) decompressed versions of the arrays $K_{p}(x, y)$ for the resolution levels $p=4, \ldots, 1$, respectively; (f) array $K(x, y)$ with the joint contribution of all the $p q$ channels; $(\mathrm{g})$ binary output image $B(x, y)$. 


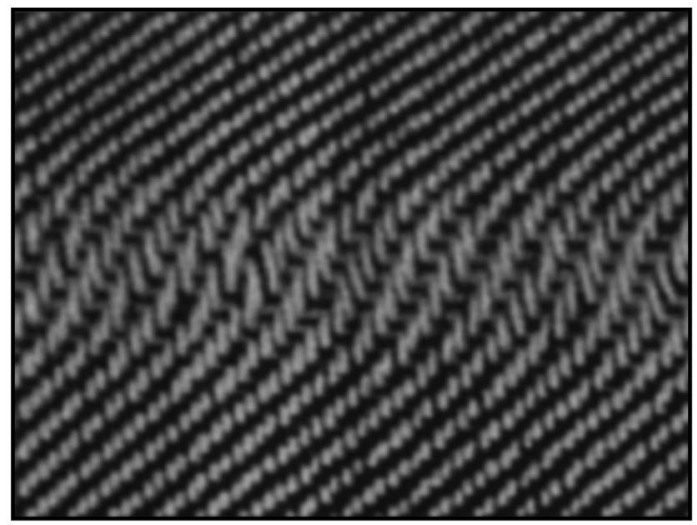

(a)

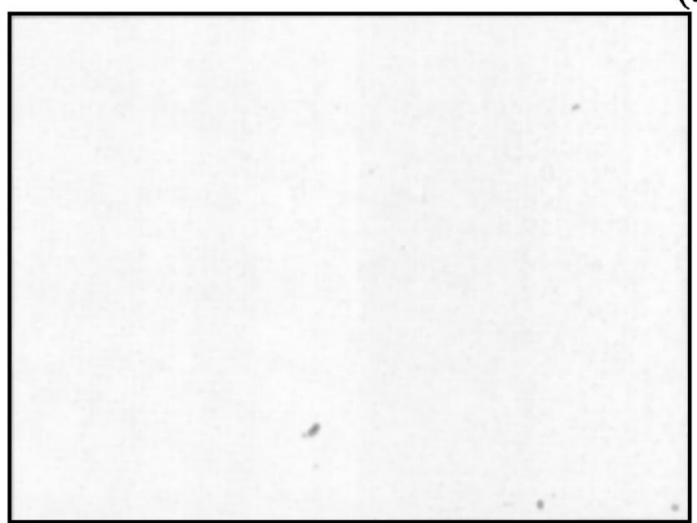

(b)

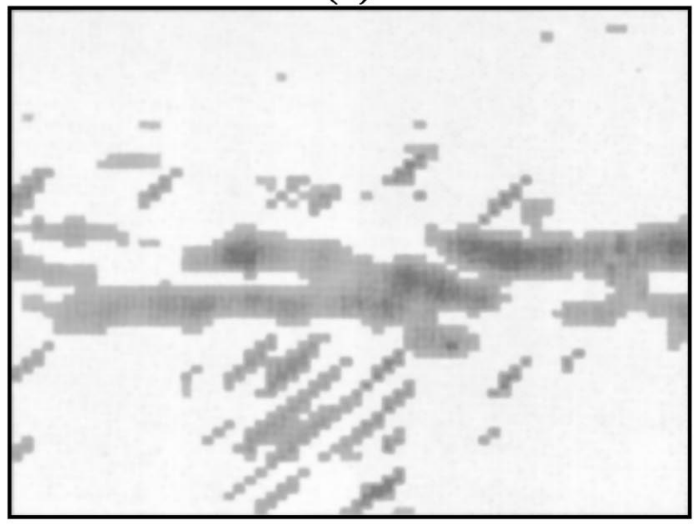

(d)

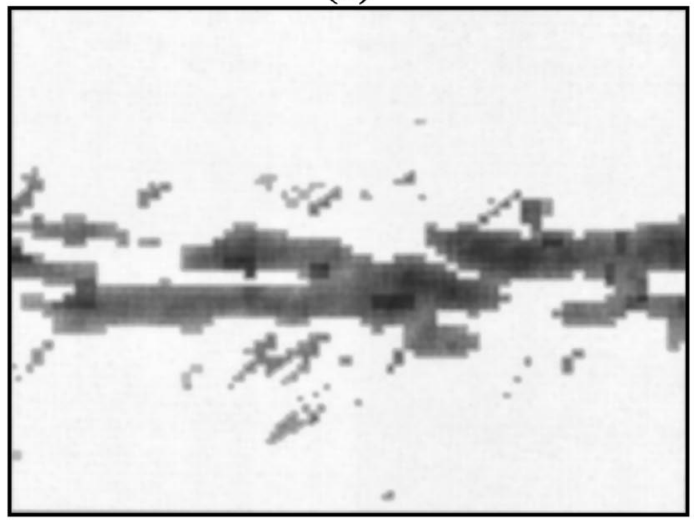

(f)

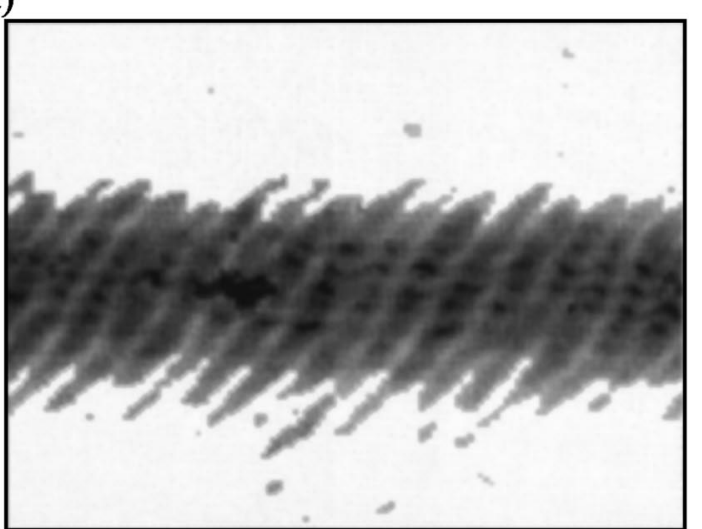

(c)

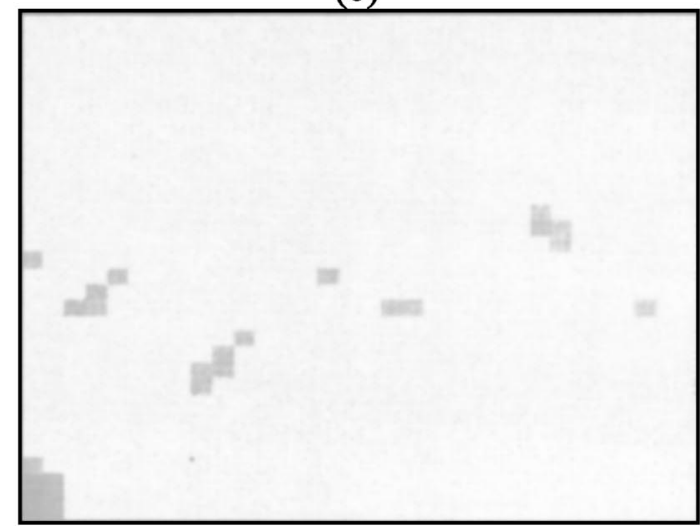

(e)

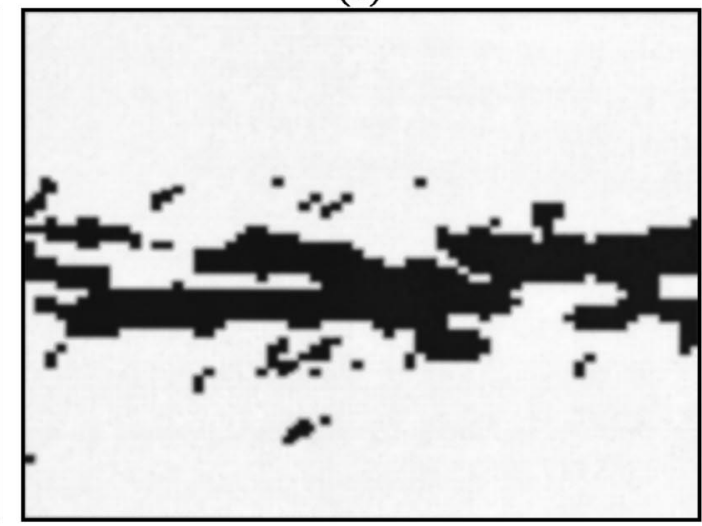

(g)

Fig. 4 (a) Thin-place effect in a twill fabric; (b) to (e) decompressed versions of the arrays $K_{p}(x, y)$ for the resolution levels $p=4, \ldots, 1$, respectively; (f) array $K(x, y)$ with the joint contribution of all the $p q$ channels; (g) binary output image $B(x, y)$. 


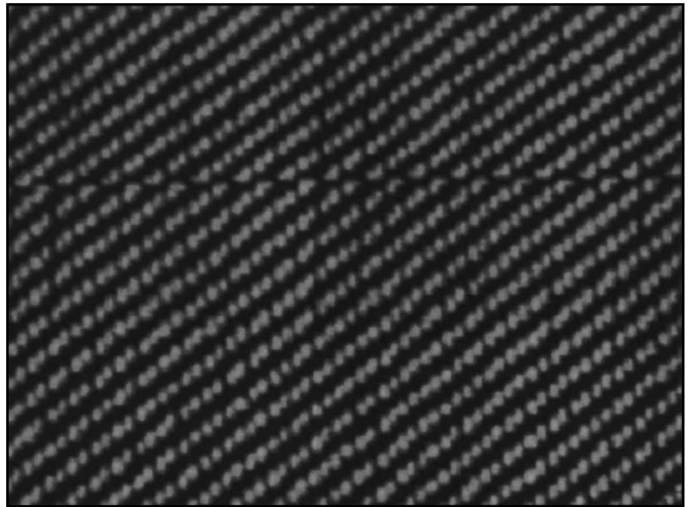

(a)

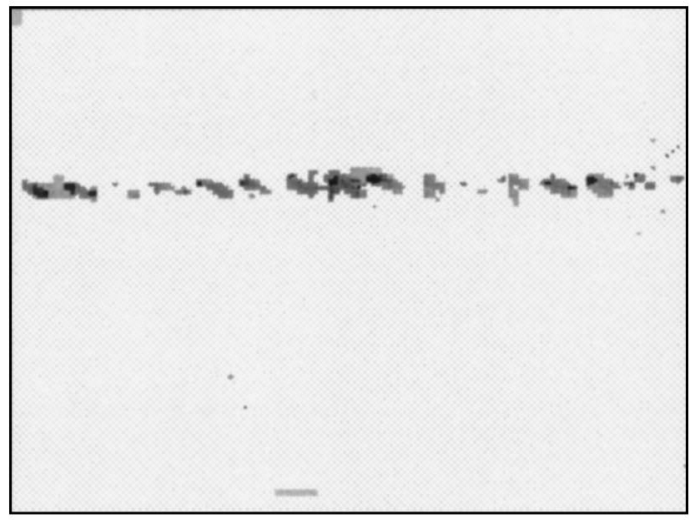

(b)

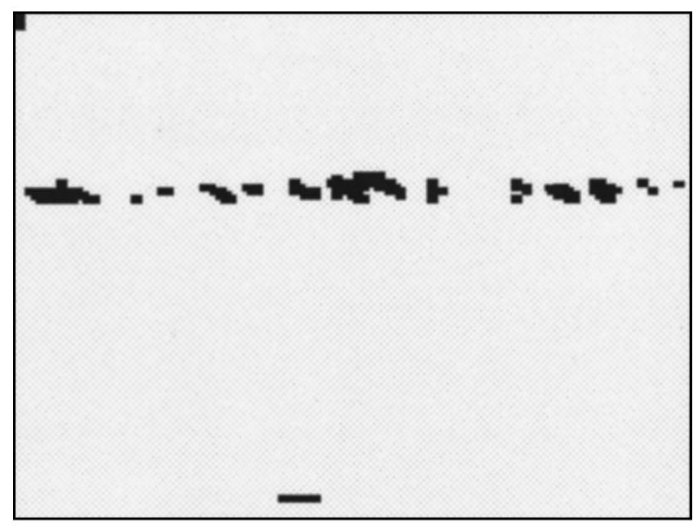

(c)

Fig. 5 (a) Twill fabric with missing yarn (mispick); (b) $K(x, y)$; (c) output image $B(x, y)$.

Finally, Fig. 10 contains a fabric with yarns of the same color. The defect is due to the crossed breaking of some yarns in both the warp and weft directions. After processing, the defect in the two perpendicular directions is correctly segmented [Figs. 10(b) and 10(c)].

\section{Conclusions}

The method proposed for local-defect detection has been shown to be a useful tool for inspecting industrial materials

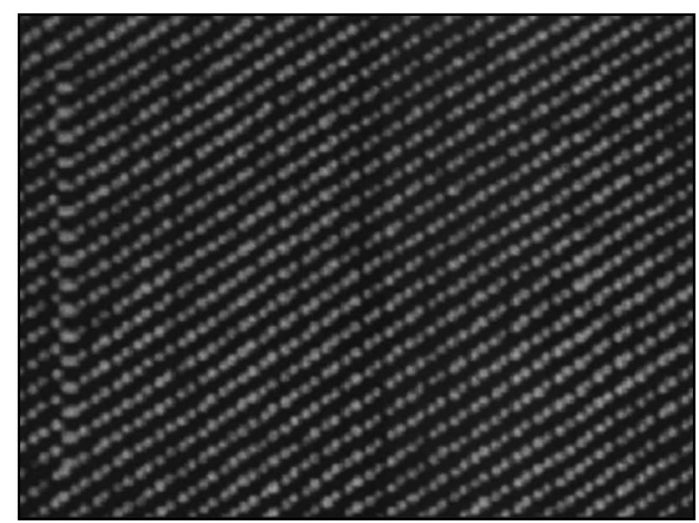

(a)

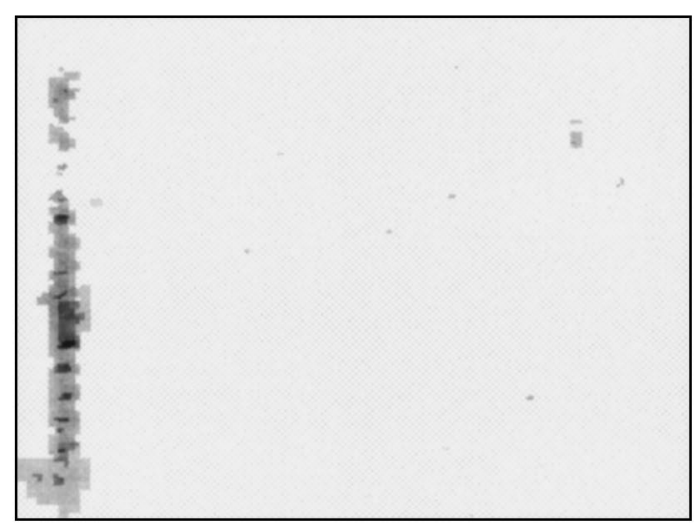

(b)

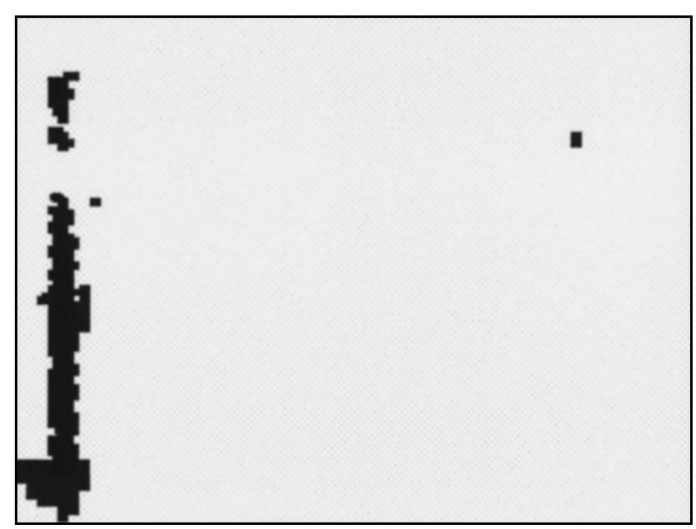

(c)

Fig. 6 (a) Twill fabric with double yarn defect; (b) $K(x, y)$; (c) output image $B(x, y)$.

with periodic regular texture. The method is based on a multiscale and multiorientation Gabor filter scheme that roughly imitates the early human vision process.

As we intended, a general improvement and enlargement of the vision system capabilities can be achieved by using the proposed algorithm to detect local defects in regular textures. Versatility, full automatism, computational efficiency, robustness, and industrial applicability were the pursued properties of the method, and we have demon- 


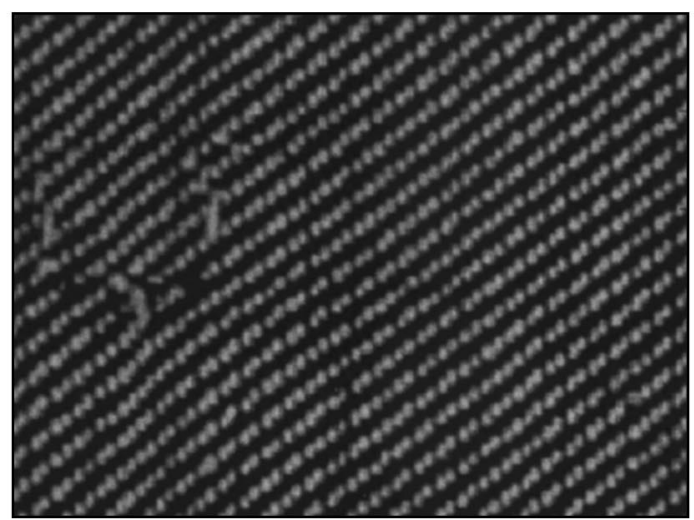

(a)

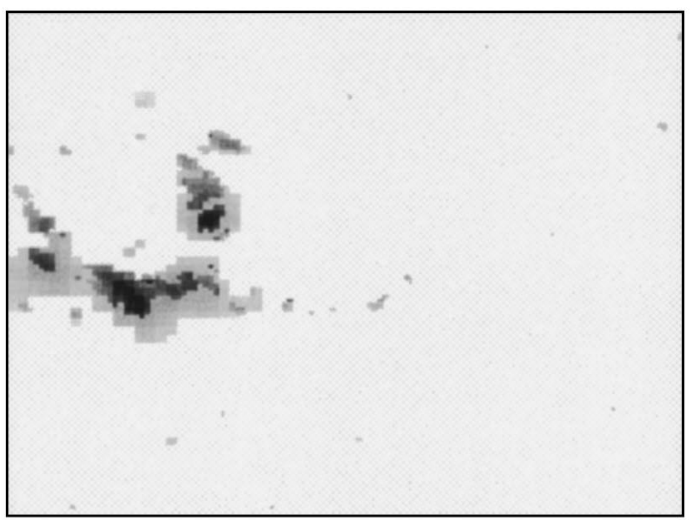

(b)

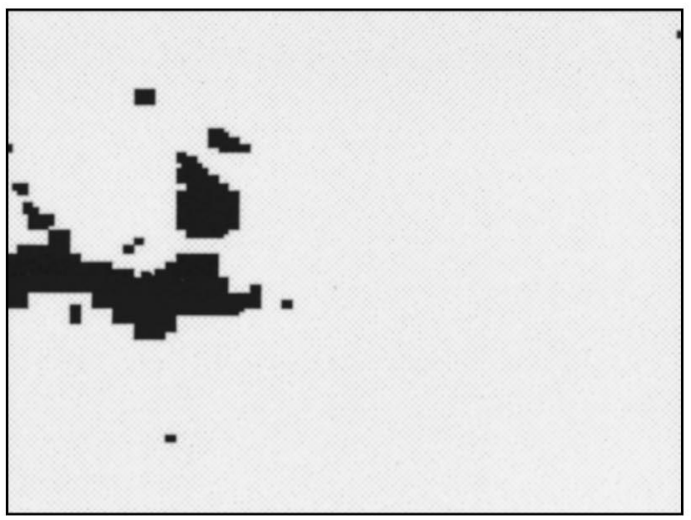

(c)

Fig. 7 (a) Twill fabric with broken yarns; (b) $K(x, y)$; (c) output image $B(x, y)$.

strated them through a selection of results obtained from textile inspection.

We have built an algorithm for the automatic application of the method to an input image of the sample under inspection. The algorithm applies the Gabor filter scheme in the spatial domain following a fast pyramid implementation for computational efficiency. An image with the joint contribution of the complete set of multiresolution and multi-orientation channels is binarized. In the binary output

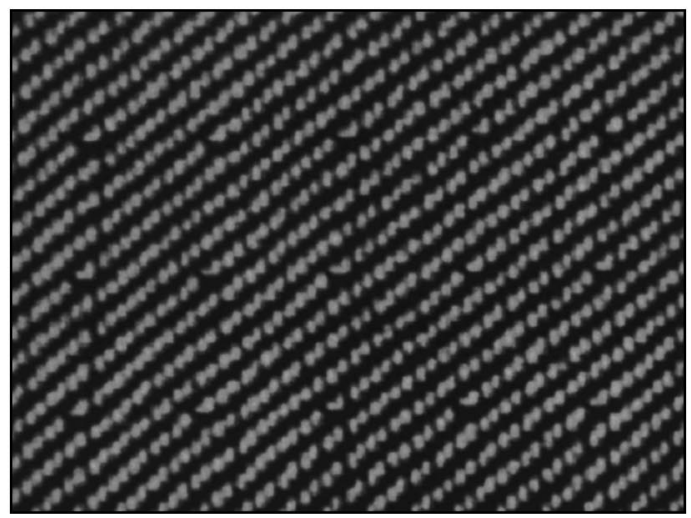

(a)

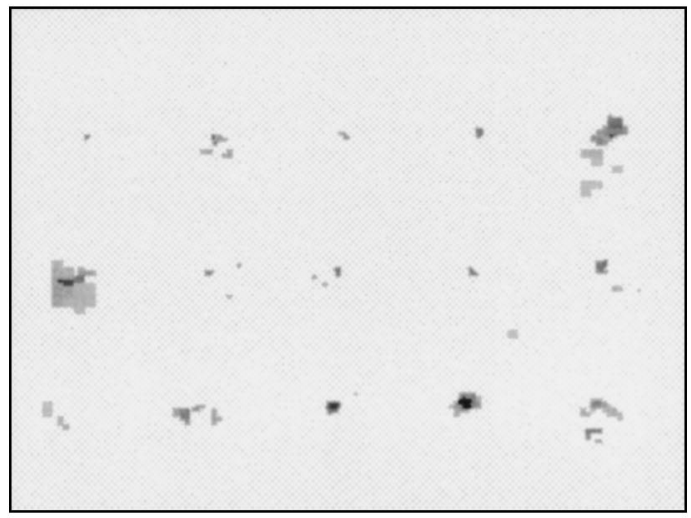

(b)

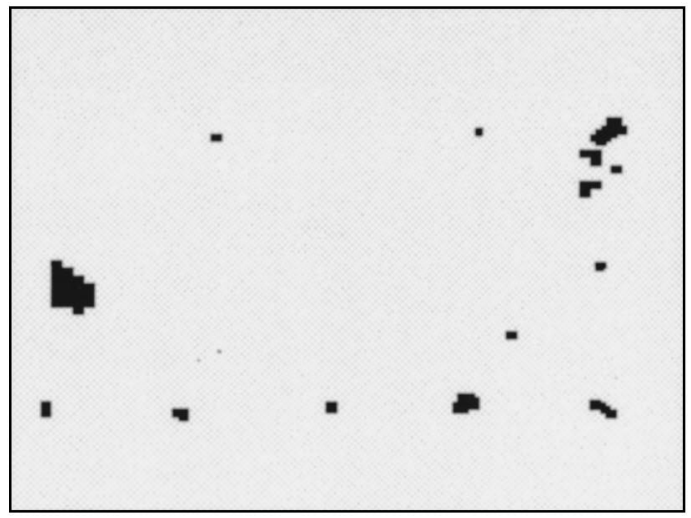

(c)

Fig. 8 (a) Twill fabric with down heddle defect; (b) $K(x, y)$; (c) output image $B(x, y)$

image local defects appear segmented from the background.

One of the most important advantages of the method is that it is multipurpose without requiring any adjustment. The only considerations that require attention are optical conditions such as lightness and scale to guarantee optimal performance, and a preliminary analysis of a prototype defect-free sample to extract the mean and standard deviation of its texture descriptors. We have avoided the use of 


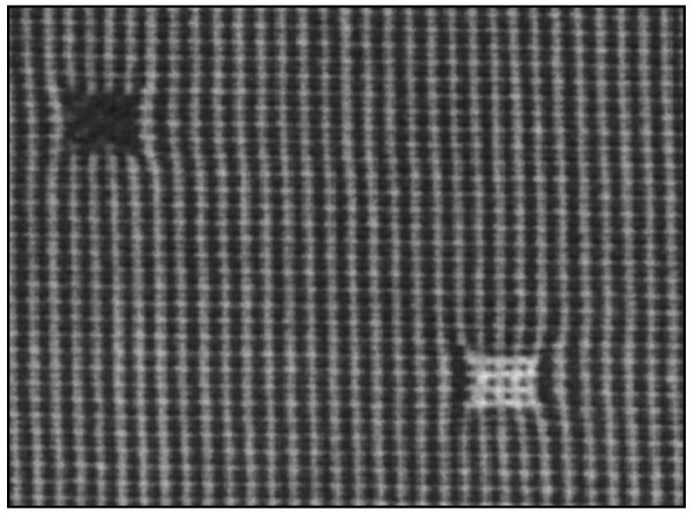

(a)

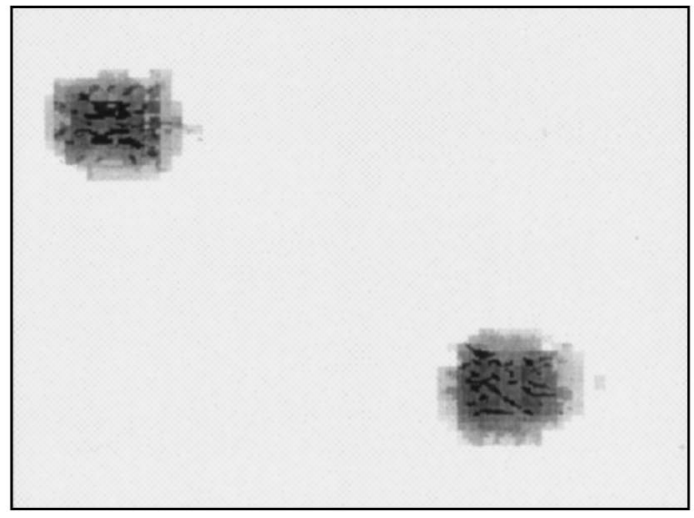

(b)

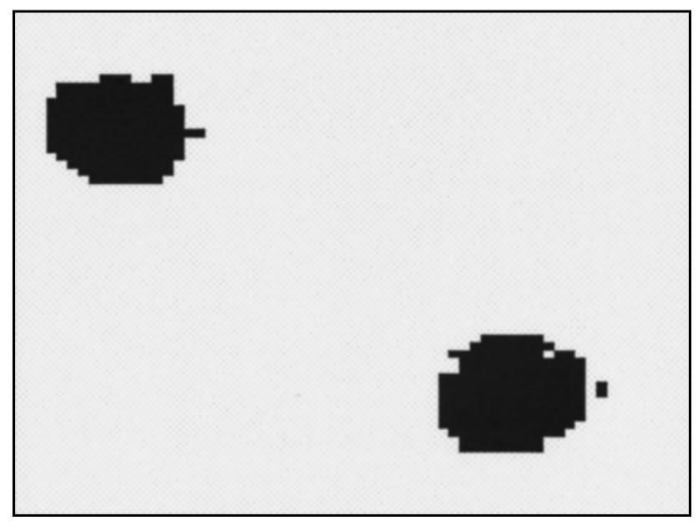

(c)

Fig. 9 (a) Large defects of different colors in a plain fabric with black and white threads; (b) $K(x, y)$; (c) output image $B(x, y)$.

adjustable weighting functions or parameters that might make the inspection process too dependent on adjustment to a particular reduced set of textures or defects. The method is robust. It is resistant to common input variations such as changes of illumination. It works with contrast rather than luminance units, and therefore it should work well under reasonable changes of brightness level. Furthermore, it can be applied to composite patterns with elements of different brightness without any particular adaptation. In

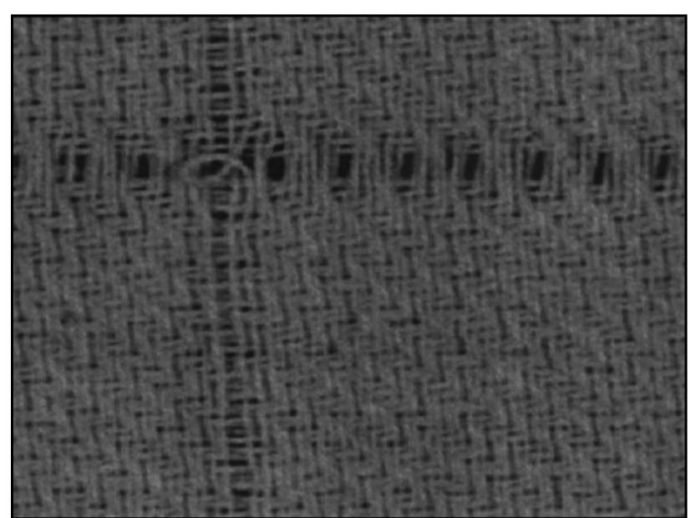

(a)

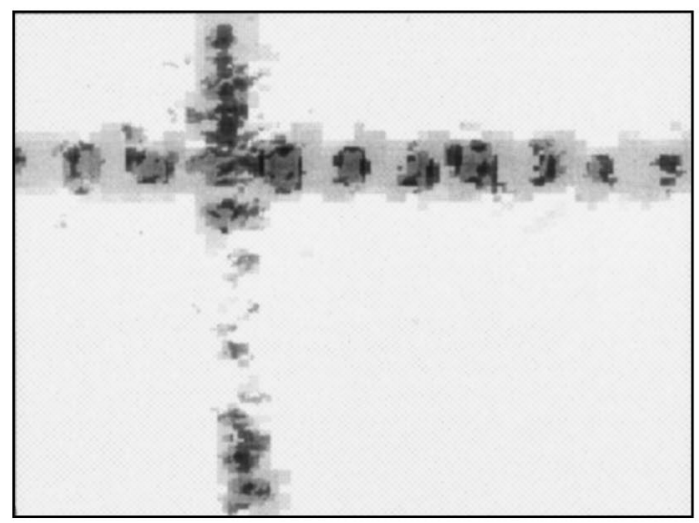

(b)

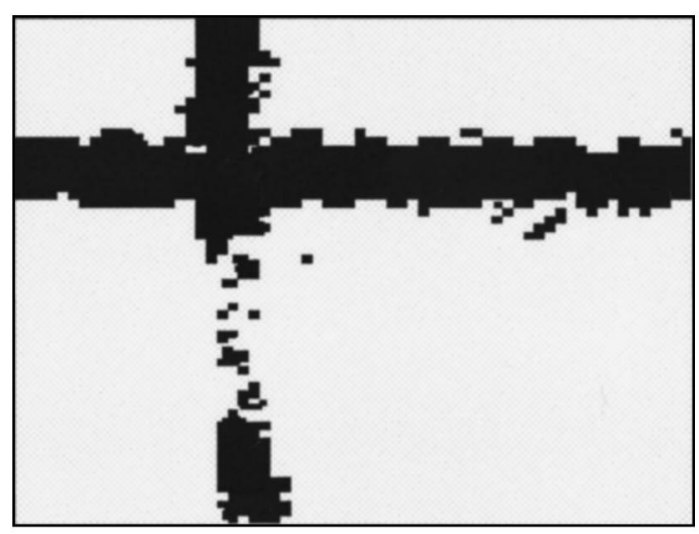

(c)

Fig. 10 (a) White twill fabric with crossed break of multiple threads; (b) $K(x, y)$; (c) output image $B(x, y)$.

addition, there is no preferred orientation in which the texture has to be fixed before applying the method.

In this paper, we have applied this multipurpose method to web inspection and noted its capabilities in detecting common local defects in woven fabrics. Although Jasper et al. ${ }^{6}$ did not rely on the capability of Gabor filters to detect local defects, we have demonstrated in this work that they actually can. This fact confirms our preliminary results previously reported. $^{7}$ 
We have tested the proposed method on a wide variety of defective fabric samples, obtaining, in general, very good results. We have presented several representative cases where different shapes, structures, colors, sizes, etc. of defects and textured background have been correctly segmented.

The versatility of the method has been demonstrated not only by its applicability to different regular textures but also, for a given texture, by its detecting a variety of defects. The method does not need human supervision or previous knowledge about the texture or defect. In fabric inspection, for example, it does not need information on the repeat pattern, in contrast with the method proposed in Ref. 6.

The results of defect detection in fabrics shown and discussed in this paper lead - as a first application-to textile inspection. Except for minor adaptations to each particular case, the method is ready to be used in an on-line industrial inspection system.

\section{Acknowledgments}

This research was supported by the Spanish CICYT under projects TAP96-1015-C03-02 and TAP97-1089 to J. Escofet, M. S. Millán, and J. Pladellorens, and grant TIC940849 to R. Navarro. We thank Professors R. Comas and X. Capdevila for their assistance with the web samples.

\section{References}

1. E. J. Wood, "Applying Fourier and associated transforms to pattern characterization in textiles," Text. Res. J. 60, 212 (1990),

2. S. A. Hosseini-Ravandi, "Fourier transform analysis of plain weave fabric appearance," Text. Res. J. 65(11), 676-683 (1995).

3. C. Ciamberlini, F. Francini, G. Longobardi, P. Sansoni, and B. Tiribilli, "Defect detection in textured materials by optical filtering with structured detectors and self-adaptable masks," Opt. Eng. 35(3), 838844 (1996)

4. M. S. Millán, and J. Escofet, "Fourier domain based angular correlation for quasiperiodic pattern recognition. Applications to web inspection," Appl. Opt. 35(31), 6253-6260 (1996).

5. W. J. Jasper and H. Potlapalli, "Image analysis of mispicks in woven fabric," Text. Res. J. 65(1), 683-692 (1995).

6. W. J. Jasper, S. J. Garnier, and H. Potlapalli, "Texture characterization and defect detection using adaptive wavelets," Opt. Eng. 35(11), 3140-3149 (1996).

7. J. Escofet, R. Navarro, M. S. Millán, and J. Pladellorens, "Detection of local defects in textile webs using Gabor filters," in Vision Systems: New Image Processing Techniques, Ph. Réfrégier, Ed., Proc. SPIE 2785, 163-170 (1996)

8. D. Gabor, "Theory of communication," J. Inst. Elect. Eng. 93, 429457 (1946).

9. J. G. Daugman, "Uncertainty relation for resolution in space, spatialfrequency and orientation optimized by two-dimensional visual cortical filters," J. Opt. Soc. Am. 2(7), 1160-1169 (1985).

10. R. Navarro, A. Tabernero, and G. Cristóbal, "Image representation with Gabor wavelets and its applications," in Advances in Imaging and Electronic Physics, Vol. 97, P. Hawkes, Ed., Academic Press (1996).

11. M. R. Turner, "Texture discrimination by Gabor functions," Biol. Cybern. 55, 71-82 (1986).
12. I. Fogel and D. Sagi, "Gabor filters as texture discriminator," Biol. Cybern. 61, 103-113 (1989).

13. T. Randen and J. Hakon-Husoy, "Multichannel filtering for image texture segmentation," Opt. Eng. 33(8), 2617-2625 (1994).

14. T. P. Weldon, W. E. Higgins, and D. F. Dunn, "Gabor filter design for multiple texture segmentation," Opt. Eng. 35(10), 2852-2863 (1996).

15. O. Nestares, R. Navarro, J. Portilla, and A. Tabernero, “Automatic computation of the area irradiated by ultrashort laser pulses in $\mathrm{Sb}$ materials through texture segmentation of TEM images," Ultramicroscopy 66, 101-115 (1996).

16. D. P. Casasent, J. S. Smokelin, and A. Ye, "Wavelet and Gabor transforms for detection," Opt. Eng. 31(9), 1893-1898 (1992).

17. R. Navarro and A. Tabernero, "Gaussian wavelet transform: two alternative fast implementations for images," Multidim. Syst. Signal Proc. 2, 421-436 (1991).

18. J. Portilla, R. Navarro, and O. Nestares, "Texture synthesis-byanalysis method based on a multiscale early-vision model," Opt. Eng. 35(8), 2403-2417 (1996).

19. W. K. Pratt, Digital Image Processing, Wiley-Interscience, p. 443 (1991).

Jaume Escofet received his MS degree in 1980 from the Universitat Autònoma de Barcelona. Since 1990 he has been professor of optics in the Department of Optics and Optometry at the Universitat Politècnica de Catalunya (Spain). He is currently working toward his $\mathrm{PhD}$ degree in the fields of digital and optical image processing, especially in textile applications.

Rafael Navarro received the MS and PhD degrees in physics from the University of Zaragoza, Spain, in 1979 and 1984, respectively. From 1985 to 1986 he was an optical and image-processing engineer at the Instituto de Astrofísica de Canarias. He joined the Instituto de Optica of the Consejo Superior de Investigaciones Científicas in 1987, where he is currently a senior scientific researcher. Since 1988 he has headed the Imaging \& Vision Group, and since 1994 he has been associate director of the Instituto de Optica. He is interested in human vision, optics, and image processing.

María S. Millán received the BS and MS degrees in physics from the Universitat de València in 1984 and 1985, respectively. In 1990 she received the $\mathrm{PhD}$ degree in physics from the Universitat $\mathrm{Au}$ tònoma de Barcelona. Since 1984 she has been a professor in the Department of Optics and Optometry of the Universitat Politècnica de Catalunya. Her fields of research involve pattern recognition, color, texture analysis, optical processing, and image processing. She is member of the Sociedad Española de Optica, the European Optical Society, and SPIE.

Josep Pladellorens received his MS degree from the Universitat de Barcelona in 1983, and his PhD in physics from the Universitat Autonoma de Barcelona in 1992. Since 1984 he has been a professor of physics in the School of Optics and Optometry of the Universitat Politècnica de Catalunya, and since 1995 director of the School. His main interests are in the field of digital image processing, both theoretical and applied. Particularly he is interested in medical imaging, stereoscopic imaging, and industrial quality control through digital imaging, especially in the textile, paper, and ophthalmic lenses industries. 